\title{
N-3 polyunsaturated fatty acids do not influence the efficacy of dual antiplatelet therapy in stable angina pectoris patients after percutaneous coronary intervention
}

Katarzyna Mizia-Stec ${ }^{1}$, Magdalena Mizia ${ }^{1}$, Maciej Haberka ${ }^{2}$, Bartosz Lasota ${ }^{2}$, Klaudia Gieszczyk-Strózik ${ }^{1}$, Artur $\mathrm{Chmiel}^{2}$, Jacek Najda ${ }^{3}$, Zbigniew Gąsior ${ }^{2}$, Sławomira Kyrcz-Krzemieńn

${ }^{1} 1^{\text {st }}$ Department of Cardiology, Medical University of Silesia, Katowice, Poland

${ }^{2}$ Department of Cardiology, Medical University of Silesia, Katowice, Poland

${ }^{3}$ Department of Hematology and Bone Marrow Transplantation, Medical University of Silesia, Katowice, Poland

\section{Abstract}

Background: We aimed to prospectively assess the influence of the recommended dose, $1.0 \mathrm{~g}$ of polyunsaturated fatty acids (N-3 PUFA) daily, on platelet reactivity in patients with stable angina pectoris (SAP) after elective percutaneous coronary intervention (PCI).

Methods: Forty consecutive patients with SAP and successful PCI were randomized to the study group (group PUFA: $n=20$; age $65 \pm 8$; standard therapy +75 mg acetylsalicylic acid $+75 \mathrm{mg}$ clopidogrel $+N-3$ PUFA/Omacor $1 \mathrm{~g}$ daily) and the control group (group $C: n=20$; age $65 \pm 9$; standard therapy $+75 \mathrm{mg}$ acetylsalicylic acid $+75 \mathrm{mg}$ clopidogrel). Platelet reactivity tests (COL, TRAP, ASPI, ADP) were performed using whole blood aggregometry (multiplate platelet [PLT] function analysis) on the $2^{\text {nd }}$ and $30^{\text {th }}$ day after PCI.

Results: Baseline patients' characteristics and clinical outcomes were comparable between the groups. There were no differences between both groups in the mean values of the PTL tests measured 30 days after PCI (PUFA vs. C: ASPI: $18.5 \pm 17$ vs. $27 \pm 29$ U, COL: $30.4 \pm 14.3$ vs. $30.3 \pm 13.4 U, A D P: 25.4 \pm 16.1$ vs. $20 \pm 10.7 U, T R A P: 65.8 \pm 25.6$ vs. $57.1 \pm 20.4 U$, $p=N S)$. The mean delta values of the PTL tests (18-24 h post-PCI/30 days post-PCI) were also comparable between the groups. The PTL aggregometry results were related to time - the baseline values of the ADP $(p=0.003), C O L(p=0.037)$ and TRAP $(p<0.001)$ tests were smaller and the ASPI $(p=0.027)$ test was higher than those measured after 1-month.

Conclusions: N-3 PUFA supplementation does not affect the efficacy of dual antiplatelet therapy in patients with SAP after PCI. (Cardiol J 2013; 20, 5: 478-485)

Key words: N-3 polyunsaturated fatty acids, antiplatelet therapy, aggregometry, percutaneous coronary intervention

\section{Introduction}

Dual antiplatelet therapy with acetylsalicylic acid (ASA) and clopidogrel (CLO) is recommended after percutaneous coronary intervention (PCI) with stent implantation to reduce the risk of instent thrombosis $[1,2]$. Despite dual antiplatelet therapy, some patients experience thrombotic

Address for correspondence: Katarzyna Mizia-Stec, $\mathrm{MD}, \mathrm{PhD}, 1^{\text {st }}$ Department of Cardiology, Medical University of Silesia, ul. Ziołowa 45/47, 40-635 Katowice, Poland, tel/fax: + 4832359 8890, e-mail: kmizia@op.pl 
cardiac events. Thus, agents that reduces the risk of thrombotic events without increasing the risk of bleeding merits further evaluation.

$\mathrm{N}-3$ polyunsaturated fatty acids (N-3 PUFA) supplementation is well-established and widely used in the primary and secondary prevention of cardiovascular diseases [2, 3]. It was demonstrated that N-3 PUFA may affect the coagulation, but in higher doses compared to the standard supplementation dose $[4,5]$. Most studies evidenced their antithrombotic effects [6-9] with no excess in clinically significant bleeding risk [10-12]. The available studies provide inconsistent results on antiplatelet N-3 PUFA action [13-15]. According to the recently published data, a short - 1-month N-3 PUFA therapy — significantly improved platelet response to clopidogrel after PCI [16, 17]. As fish oil consumption and N-3 PUFA supplementation are recommended for the secondary prevention of cardiovascular events [2], the potential antiplatelet effects would be of great importance for patients on dual antiplatelet therapy.

There are several platelet (PTL) function tests available. Whole blood impedance aggregometry (WBA) is one of the new methods with a high sensitivity for antiplatelet drugs (ASA, CLO, Prasugrel, IIb/IIIa antagonist). WBA may be a reliable tool in the assessment of N-3 PUFA and ASA/CLO interactions, but the evidence is scarce [18]. WBA is based on PTL activation stimulated in vitro with different agonists: arachidonic acid (AA) in the ASPI test, collagen in the COL test, ADP in the ADP test and a thrombin receptor activating peptide (TRAP-6) in the TRAP test. PLT response to CLO is well evaluated by the ADP and indirectly by the TRAP tests. The assessment of ASA antiplatelet effectiveness is measured using the ASPI and COL tests.

Reassuming, there are also some data on N-3 PUFA induced PTL inhibition in the literature. The daily doses of N-3 PUFA administered in most studies were high and therefore the influence of the standard recommended dose of $1.0 \mathrm{~g}$ daily is of great interest, especially because WBA as a laboratory PTL function assay is now available and may serve as a useful tool in the evaluation of PTL-N-3 PUFA interactions. Thus, the objective of the study was to assess the influence of N-3 PUFA therapy on PLT reactivity to ASA and CLO measured using WBA in patients with stable angina pectoris (SAP) after PCI.

\section{Methods}

The study population consisted of 40 consecutive patients with SAP (Canadian Cardiovascular
Society [CCS] I-III) and successful PCI with stent (bare metal stent [BMS] or drug eluting stent [DES]) implantation. The standard pharmacotherapy used before the study enrollment was similar for both groups, including ASA $(75 \mathrm{mg}$ daily) for at least 3 months prior to the study and CLO with loading dose $(600 \mathrm{mg})$ in the day of PCI procedure followed by daily doses of $75 \mathrm{mg}$ during the study. The patients were randomized into two study groups: group PUFA (female/male $=8 / 12$; age $65 \pm 8$ ) with N-3 PUFA (Omacor 1 g daily) supplementation started $24 \mathrm{~h}$ after CLO loading dose and the control group matched as to age and sex (female/male $=6 / 15$; age $65 \pm 90$ ) (Fig. 1). Standard pharmacotherapy following the European Society of Cardiology (ESC) recommendations was given to all of the patients: ASA (75 mg/d), CLO $(75 \mathrm{~m} / \mathrm{d})$, statin, beta-blocker and angiotensin converting enzyme-inhibitors (ACE-I) were adjusted to heart rate and blood pressure.

The exclusion criteria included: acute coronary syndrome (ACS), cardiac troponin I concentration $>0.01 \mathrm{ng} / \mathrm{mL}$, a history of myocardial infarction (MI) within 6 months prior to the enrollment into the study, acute and chronic inflammatory diseases (in the 3 preceding months), $2^{\text {nd }}$ and $3^{\text {rd }}$ degree of hypertension according to the ESC guidelines, myocarditis and vasculitis, current gastrointestinal tract diseases, diseases of the aorta, hormone replacement therapy, spondyloarthritis, hematological disorders, underlying malignancies, comorbid psychiatric or neurological disorders, treatment with GP IIb/IIIa inhibitors, oral anticoagulant therapy, acute and chronic kidney disease (glomerular filtration rate $<60 \mathrm{~mL} / \mathrm{min} / 1.73 \mathrm{~m}^{2}$ ), use of nonsteroid anti-inflammatory drugs and steroids.

All the patients gave the written informed consent, and our study protocol was approved by our institution's human research committee, conformed to the ethical guidelines by the 1975 Declaration of Helsinki.

All of the subjects had a clinical assessment comprising a detailed medical history (family history, previous ST/non-ST elevation myocardial infarction [STEMI/NSTEMI], previous coronary artery by-pass grafting [CABG], concomitant diseases, smoking status, pharmacotherapy) and a physical examination. Anthropometric measurements were obtained and body mass index (BMI) and waist to hip ratio (WHR) were calculated. The standard diagnostic tests (ECG, TTE) were done. Echocardiography was performed in all patients according to the guidelines of the European Society of Echocardiography. PCI was performed using the 


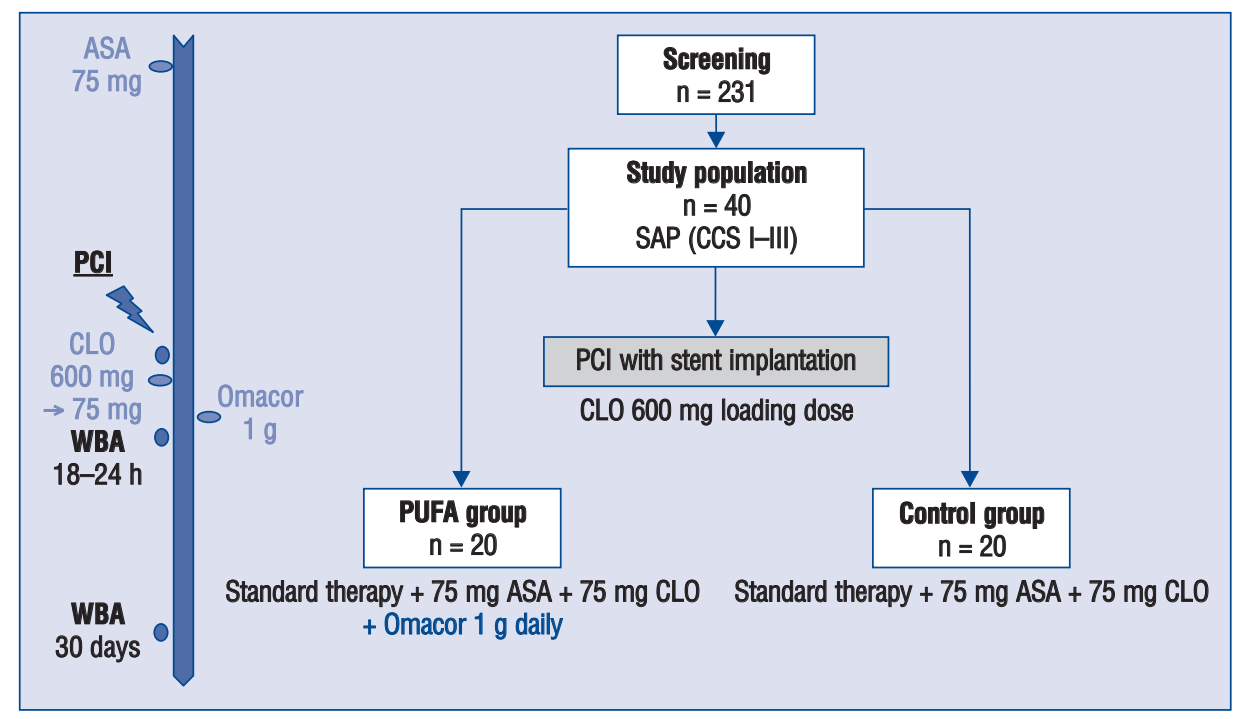

Figure 1. Participant flow; ASA — acetylsalicylic acid; CLO — clopidogrel; $\mathrm{PCl}$ - percutaneous coronary intervention; SAP — stable angina pectoris; WBA — whole blood impedance aggregometry.

standard protocols. Clinical data (CCS class, incidence of unstable angina, MI, re-PCI, CABG, death) as well as compliance with the study drug administration was verified at the 1-month follow-up.

Blood samples were obtained in the morning after fasting from the cubital vein in supine patients into $5-10 \mathrm{~mL}$ blood collection tubes using venipuncture. Blood morphology was assessed manually in a local laboratory at hospital admission.

Platelet function analysis was performed using the Multiplate analyzer - Multiple Electrode Aggregometry (MEA) and a point-of-care whole blood impedance aggregometer (Dynabate, Munich, Germany). The baseline PTL function analysis was assessed 18-24 h after the PCI + CLO loading dose (600 $\mathrm{mg}$ ) and before administration of N-3 PUFA. The second sample was obtained 30 days after PCI (standard therapy as was mentioned earlier). In the impedance aggregometry method, resting blood platelets are activated by particular agonists, expose receptors and attach to the test surfaces. The analyzer continuously measures the resistance which correlates with the platelets adhering to the electrodes. The procedure includes $300 \mu \mathrm{L}$ whole blood and $300 \mu \mathrm{L}$ saline $0.9 \%$, preheated at $37^{\circ} \mathrm{C}$ and incubated for $180 \mathrm{~s}$, afterwards $20 \mu \mathrm{L}$ of the particular agonist solution is added and the test is performed (6 min). The final concentration of the test solutions were as follows: ADP $6.5 \mu \mathrm{M}$ (ADP test), COL $3.2 \mu \mathrm{g} / \mathrm{mL}$ (COL test), AA $0.5 \mathrm{mM}$ (ASPI test), TRAP $32 \mu \mathrm{M}$ (TRAP test). The analyzer has 5 cells for parallel testing.

WBA was performed with the same kit to avoid inter-kit variability.

\section{Statistical analysis}

All text and table results are expressed as means \pm standard deviation (SD) for normally distributed variables and as medians for abnormally distributed variables or as a number and percentage. The results' normal distribution was analyzed using the Kolmogorov-Smironov test. Baseline clinical parameters and the results of ancillary investigations were compared using the to-sample t-tests for normally distributed continuous variables (Student's t-test); in the case of abnormal distribution, the Mann-Whitney U test was used. To compare the change of PTL function tests over time, the data were analyzed as repeated measures analysis of variances, taking into account intervention factors and time. The Spearman rank model was used for correlation analysis of the baseline (PTL tests results - data obtained at 1 day after PCI) parameters. The Pearson model was used to determine the relationships between the delta values of the variables (18-24 $\mathrm{h}$ post-PCI/30 days post-PCI). Multivariable linear regression was used to assess independent predictors of the PTL function tests as well as the delta values of the variables. A value $p<0.05$ was considered statistically significant.

\section{Baseline parameters}

\section{Results}

There were no significant differences between both groups in baseline demographics (PUFA: $\mathrm{n}=20$; age: $65 \pm 8.5 ; \mathrm{F} / \mathrm{M}: 8 / 12 ; \mathrm{BMI}: 29.6 \mathrm{~kg} / \mathrm{m}^{2}$; WHR 0.98 vs. C: $\mathrm{n}=20$; age: $64 \pm 8.4$, F/M: 6/14; BMI: $29.3 \mathrm{~kg} / \mathrm{m}^{2}$; WHR: 0.95; $\mathrm{p}=\mathrm{NS}$ ), medical 
Table 1. Group PUFA and group $C$ baseline characteristics.

\begin{tabular}{|c|c|c|c|}
\hline & Group PUFA (n = 20) & Group C $(n=20)$ & $\mathbf{P}$ \\
\hline Age [years] & $65 \pm 8.5$ & $64.4 \pm 8.4$ & NS \\
\hline Female/male & $8(40 \%) / 12(60 \%)$ & $6(30 \%) / 14(70 \%)$ & NS \\
\hline Body mass index $\left[\mathrm{kg} / \mathrm{m}^{2}\right]$ & $29.6 \pm 3.8$ & $29.3 \pm 4.2$ & NS \\
\hline Waist-to-hip ratio & $0.98 \pm 0.09$ & $0.95 \pm 0.11$ & NS \\
\hline Hypertension & $18(90 \%)$ & $17(85 \%)$ & NS \\
\hline Hyperlipidemia & $18(90 \%)$ & $16(90 \%)$ & NS \\
\hline Diabetes mellitus & $7(35 \%)$ & $6(30 \%)$ & NS \\
\hline Current smoking & $5(25 \%)$ & $7(35 \%)$ & NS \\
\hline STEMI/NSTEMI in medical history & $7(35 \%) / 1(5 \%)$ & $8(40 \%) / 0(0 \%)$ & NS \\
\hline CABG in medical history & $0(0 \%)$ & $2(10 \%)$ & NS \\
\hline Total cholesterol [mg/dL] & $164.8 \pm 48.1$ & $171 \pm 71$ & NS \\
\hline Low density lipoprotein [mg/dL] & $91.4 \pm 41.2$ & $93.2 \pm 51$ & NS \\
\hline High density lipoprotein [mg/dL] & $39.4 \pm 19.3$ & $35.5 \pm 12.3$ & NS \\
\hline Triglicerydes [mg/dL] & $147.3 \pm 64.3$ & $162 \pm 71.4$ & NS \\
\hline Red blood cells $\left[\times 10^{6} / \mu \mathrm{L}\right]$ & $4.5 \pm 0.5$ & $4.7 \pm 0.3$ & NS \\
\hline White blood cells $\left[\times 10^{3} / \mu \mathrm{L}\right]$ & $7.3 \pm 2.5$ & $6.8 \pm 1.2$ & NS \\
\hline Creatinine $[\mathrm{mg} / \mathrm{dL}]$ & $0.9 \pm 0.3$ & $0.9 \pm 0.2$ & NS \\
\hline End-diastolic diameter [mm] & $50.6 \pm 4.5$ & $48.3 \pm 6.3$ & NS \\
\hline End-systolic diameter [mm] & $30.7 \pm 6.5$ & $32.4 \pm 5.9$ & NS \\
\hline Ejection fraction [\%] & $59.4 \pm 6.9$ & $55.5 \pm 8.7$ & NS \\
\hline
\end{tabular}

STEMI — ST elevation myocardial infarction; NSTEMI — non ST elevation myocardial infarction; CABG — coronary artery bypass graft

Table 2. Group PUFA and group $C$ baseline platelets laboratory tests.

\begin{tabular}{|c|c|c|c|c|c|}
\hline \multirow[t]{2}{*}{ Standard laboratory tests } & \multicolumn{2}{|c|}{ Group PUFA (n = 20) } & \multicolumn{2}{|c|}{ Group $C(n=20)$} & \multirow[t]{2}{*}{$\mathbf{P}$} \\
\hline & Mean \pm SD & $\begin{array}{c}25 / 75 \\
\text { percentile }\end{array}$ & Mean \pm SD & $\begin{array}{c}25 / 75 \\
\text { percentile }\end{array}$ & \\
\hline Platelets $\left(\times 10^{3} / \mu \mathrm{L}\right)$ & $168.4 \pm 59.9$ & $118.5 / 223$ & $190.9 \pm 23.6$ & $174 / 202$ & NS \\
\hline Platelet distribution width [fl] & $13.1 \pm 2.5$ & $11.9 / 13.6$ & $12.3 \pm 1.5$ & $11.3 / 13.5$ & NS \\
\hline Mean platelet volume [fl] & $10.7 \pm 0.9$ & $10 / 11.8$ & $10.4 \pm 0.7$ & $10 / 10.9$ & NS \\
\hline Large platelets [\%] & $30 \pm 6.8$ & $25.5 / 32.3$ & $27.6 \pm 5.3$ & $25.5 / 32.7$ & NS \\
\hline
\end{tabular}

(PUFA: mean CCS: $2.1 \pm 0.4$; previous STEMI/ /NSTEMI: $7 / 1$; previous CABG: 0 vs. C: mean CCS: $2.2 \pm 0.6$; STEMI/NSTEMI: 8/0; previous CABG: $2 ; \mathrm{p}=\mathrm{NS}$ ) characteristics, concomitant diseases, laboratory tests (Tables 1,2 ), pharmacotherapy (Table 3), echocardiographic findings (left ventricular ejection fraction: PUFA: $59 \pm 7 \%$ vs. C: $56 \pm 9 \%$ ). Successful PCI with stent implantation (thrombolysis in myocardial infarction - TIMI 3 flow) was performed in all patients (PUFA: BMS: 18, DES: 2 vs. C: BMS: 19 vs. DES: $1 ; \mathrm{p}=\mathrm{NS}$ ).
The baseline values of PTL function tests assessed 18-24 $\mathrm{h}$ after the CLO loading dose were comparable between the groups (Table 4).

\section{Main outcomes after 1-month therapy}

The clinical outcome was comparable between the study groups; there were no differences in CCS class. No incidence of unstable angina, MI, re-PCI, urgent $\mathrm{CABG}$ or death were noted.

In the ANOVA analysis, we found no associations between the PTL test results and the group 
Table 3. Group PUFA and group C pharmacotherapy.

\begin{tabular}{lccc}
\hline & Group PUFA (n= 20) & Group C (n= 20) & P \\
\hline Acetylsalicylic acid & $20(100 \%)$ & $20(100 \%)$ & NS \\
Clopidogrel & $20(100 \%)$ & $20(100 \%)$ & NS \\
Beta-blocker & $19(95)$ & $18(90 \%)$ & NS \\
Angiotensin converting enzyme inhibitor & $20(100 \%)$ & $20(100 \%)$ & NS \\
Diuretics & $8(40 \%)$ & $12(60 \%)$ & NS \\
Statin & $20(100 \%)$ & $20(100 \%)$ & NS \\
Fibrate & $4(20 \%)$ & $8(40 \%)$ & NS \\
Oral diabetes mellitus drugs & $7(35 \%)$ & $6(30 \%)$ & NS \\
Nitroglycerin & $4(20 \%)$ & $4(20 \%)$ & NS \\
\hline
\end{tabular}

Table 4. ANOVA analysis - interaction of time and intervention in the study groups.

\begin{tabular}{|c|c|c|c|c|c|c|c|}
\hline \multirow[t]{2}{*}{ Test* } & \multicolumn{2}{|c|}{ Group PUFA $(n=20)$} & \multicolumn{2}{|c|}{ Group C $(n=20)$} & \multicolumn{3}{|c|}{ P Source of variatione } \\
\hline & Baseline & $\begin{array}{l}\text { After } \\
1 \text { month }\end{array}$ & Baseline & $\begin{array}{c}\text { After } \\
1 \text { month }\end{array}$ & $\begin{array}{c}\text { Group } \\
\text { (G) }\end{array}$ & $\begin{array}{c}\text { Time } \\
(\mathrm{T})\end{array}$ & $\begin{array}{c}\text { Interaction } \\
\mathbf{G} \times \mathrm{T}\end{array}$ \\
\hline ADP [U] & $25.5 \pm 16.0$ & $29.3 \pm 13.2$ & $20.0 \pm 10.7$ & $25.0 \pm 8.3$ & 017 & 0.003 & 0.34 \\
\hline ASPI [U] & $18.5 \pm 17.0$ & $26.9 \pm 20.7$ & $27.0 \pm 28.8$ & $23.1 \pm 13.9$ & 0.94 & 0.027 & 0.69 \\
\hline COL [U] & $30.5 \pm 14.3$ & $33.2 \pm 8.6$ & $30.3 \pm 13.4$ & $33.1 \pm 7.9$ & 0.97 & 0.037 & 0.92 \\
\hline TRAP [U] & $65.8 \pm 25.6$ & $80.1 \pm 20.7$ & $67.1 \pm 20.4$ & $85.3 \pm 23.6$ & 0.69 & $<0.001$ & 0.66 \\
\hline
\end{tabular}

*Platelets activity tests with different activators: arachidonic acid (ASPI), adenosine diphosphate (ADP), thrombin receptor activating peptide-6 (TRAP), collagen (COL).

Table 5. Comparison of delta platelet test results (baseline and 1 month after percutaneous coronary intervention).

\begin{tabular}{lcccccc}
\hline \multirow{2}{*}{ Test* } & \multicolumn{2}{c}{ Group PUFA $(\mathbf{n}=\mathbf{2 0})$} & & \multicolumn{2}{c}{ Group C $(\mathbf{n}=\mathbf{2 0})$} & P \\
\cline { 2 - 3 } & Mean \pm SD & 25-75 percentile & & Mean \pm SD & 25-75 percentile & \\
\hline Delta ADP & $3.8 \pm 12.0$ & $-3.5 / 11$ & & $5.0 \pm 10.4$ & $-6 / 12$ & 0.73 \\
Delta ASPI & $8.5 \pm 25.0$ & $-1 / 13.5$ & & $-3.9 \pm 24.1$ & $-4 / 13$ & 0.12 \\
Delta COL & $2.7 \pm 15.3$ & $-4 / 11.5$ & & $2.8 \pm 11.2$ & $-5 / 10$ & 0.98 \\
Delta TRAP & $14.3 \pm 19.7$ & $3 / 25.5$ & & $18.2 \pm 31.0$ & $-6 / 31$ & 0.63 \\
\hline
\end{tabular}

*Platelets activity tests with different activators: arachidonic acid (ASPI), adenosine diphosphate (ADP), thrombin receptor activating peptide-6 (TRAP), collagen (COL).

(PUFA or C). The PTL aggregometry results were related to the time of observation - the baseline values of the $\mathrm{ADP}(\mathrm{p}=0.003)$, COL $(\mathrm{p}=0.037)$ and TRAP $(p<0.001)$ tests were smaller and the ASPI $(p=0.027)$ test was higher than those measured after the 1-month observation (Table 4). The mean delta values of PTL tests results were also comparable between the groups (Table 5).

\section{Regression analysis}

Clinical data. The TRAP test values were correlated with patients' age $(\mathrm{r}=0.354, \mathrm{p}=0.021)$. The COL test results were correlated with HDL- -cholesterol levels $(r=-0.263, p=0.033)$ and in a borderline manner to creatinine serum levels $(r=0.229, p=0.064)$. Significant correlations between the delta PTL test results and serum creatinine levels were also found. The serum creatinine level correlated with delta ASPI and delta COL $(\mathrm{r}=0.440, \mathrm{p}=0.05$ and $\mathrm{r}=0.456, \mathrm{p}=0.046)$ in the PUFA group and with delta ADP and delta ASPI $(\mathrm{r}=-0.650, \mathrm{p}=0.007$ and $\mathrm{r}=-0.533, \mathrm{p}=0.028)$ in the group $\mathrm{C}$.

PTL count, volume and morphology. Significant correlations between the delta PTL test results and PTL count, volume and morphology 
were observed. In all patients examined, delta ADP and delta COL were correlated with the PTL count $(\mathrm{r}=-0.407, \mathrm{p}=0.008$ and $\mathrm{r}=-0.534$, $\mathrm{p}<0.001)$; the PTL count correlated with delta $\mathrm{COL}(\mathrm{r}=-0.624, \mathrm{p}=0.003)$ in the PUFA group and with delta ADP in the group $\mathrm{C}(\mathrm{r}=-0.535$, $\mathrm{p}=0.02$ ).

In the PUFA group, delta ASPI correlated with the PTL-anisocytosis $(r=0.602, p=0.005)$, PTL volume $(\mathrm{r}=0.516, \mathrm{p}=0.019)$ and PTL distribution width $(\mathrm{r}=0.547, \mathrm{p}=0.012)$.

In all patients examined there were also significant correlations between the TRAP test results and the PTL count $(r=0.409, p=0.006)$ and between the COL test values and both the mean PTL volumes $(r=0.342, \mathrm{p}=0.024)$ and the PLT distribution width $(\mathrm{r}=0.363$, $\mathrm{p}=0.018$ ).

Multivariate correlation analysis did not reveal any statistically significant associations.

\section{Discussion}

In the present study, we assessed the effect of the co-administration of the recommended dose of N-3 PUFA with dual antiplatelet therapy (ASA with CLO) on the values of the PLT function tests. The results showed that N-3 PUFA did not affect the efficacy of dual antiplatelet therapy.

The PTL reactivity assessed 18-24 h after the PCI and CLO loading dose were comparable to the results after a 1-month follow-up. The degree of PTL suppression was typical - low values of the ADP, ASPI and COL tests were observed with higher values of the TRAP test. The TRAP test mainly represents the platelet-activation of thrombin and thus constitutes a sensitive marker of IIb/IIIa antagonists; CLO only exerts a weak effect on the TRAP test [19]. The relatively high on-treatment PTL aggregation that was observed in both groups could be associated with the high prevalence of hypertension, hyperlipidemia and diabetes.

In addition, we did not observe any differences in the clinical outcome of the study groups. The supplementation did not interfere with the clinical efficacy of antiplatelet therapy. It can be concluded that N-3 PUFA supplementation in the recommended standard dose does not increase the risk for restenosis or the risk of bleeding.

There are some data on N-3 PUFA induced PTL inhibition in the literature; however, the exact mechanism of the process is not well understood [20]. The study of Larson et al. [21] showed that omega-3 fatty acids in a daily dose of $3.4 \mathrm{~g}$ may modulate collagen signaling in human platelets. Our observations did not reveal any difference in COL test results after the administration of $1.0 \mathrm{~g}$ N-3 PUFA. The study of Cohen et al. [22] revealed that escalating doses of N-3 PUFA from $1 \mathrm{~g}$ to $8 \mathrm{~g}$ daily provided an increase in mean bleeding time in a dose-dependent manner. The study was performed in subjects with no antiplatelet agent, in subjects with ASA and ASA plus CLO. Finally, the authors concluded that N-3 PUFA attenuates PTL activation even among patients taking ASA or ASA plus CLO. This was associated with an increase in the negative PTL surface charge as well as with the production of thromboxan A3 from N-3 PUFA. Thus, N-3 PUFA may exert a synergistic effect with established antiplatelet agents [22]. In the same study groups, light transmission aggregometry (LTA) did not detect any additional antiplatelet effects of N-3 PUFA, which was in agreement with our findings. We may suspect that both LTA and WBA may not be sensitive enough tools to detect subtle changes in PTL function.

There is limited data on the relationship between blood coagulation and the administration of $1.0 \mathrm{~g} \mathrm{~N}-3$ PUFA daily in the literature [4, 5, 16-18]. Most of the research did not confirm any interactions $[4,5,18]$. According to the recently published data, $1.0 \mathrm{~g}$ N-3 PUFA daily added to the standard dual antiplatelet therapy after PCI influences PTL response to CLO [16, 17]. The authors in the studies with a protocol similar to our study demonstrated both a lower P2Y12 reactivity index and adenosine induced PTL aggregation in the group treated with ASA, CLO and N-3 PUFA [16, 17]. The PTL light transmission aggregometry in PTL-poor plasma and the vasodilator-stimulated phosphoprotein test (VASP) were used, which did not allow for a reliable comparison to our findings.

High dose N-3 PUFA treatment may reduce PTL and erythrocyte cell count slightly [23]. We only assessed the baseline blood cell morphology, thus we were not able to verify any impact of $1.0 \mathrm{~g} \mathrm{~N}-3$ PUFA on these parameters.

The additional finding of the study was the relationship between the results of the PTL function tests and the clinical data. These findings are in accordance with previously published observations $[23,24]$ and suggest that the analysis of standard morphology, i.e. PTL count, may be helpful in the prediction of ASA and CLO responsiveness. Moreover, we found age, serum lipids and creatinine to be modifying factors for PTL test results. 
There are some limitations as well as advantages of the study. Our study had a relatively limited sample size; however, the well-matched study groups allowed for an objective analysis. The follow-up was only at the first month after PCI; however, 2 arguments were taken into account. Firstly, this is the most important period for the appearance of in-stent thrombosis. Secondly, standard dual antiplatelet therapy after elective PCI with BMS implantation was recommended for 4-6 weeks [1]. We did not find any differences in the end-points between the study groups, which did not allow us to establish whether N-3 PUFA influences the clinical outcomes during follow-up. This issue requires a different study protocol and a larger study group.

There are some disadvantages for all PTL function assays. Most tests are not standardized and there are no reference values available [25, 26]. Lordkipanidze et al. [27] did not observe a strong correlation between different PTL function tests. We were aware of these limitations and therefore we used one, novel test - WBA - and compared the results of the same patients at 2 points in time - before and after therapeutic intervention. WBA is a reliable method for PTL function evaluation that allows antiplatelet therapy to be monitored in clinical practice and for clinical investigations. However, we cannot exclude that certain assays may not be suitable to detect all effects on PTL function.

On the other hand, we should be aware that CLO co-prescription makes it very difficult to specifically evaluate the aspirin response, since P2Y12-inhibitors affect aspirin's inhibition of arachidonic acid-induced PTL aggregation [28, 29].

According to the recently published data, we should also take into account the antithrombotic effects of N-3 PUFA supplementation in patients after PCI in stable CAD. It was demonstrated that N-3 PUFA decreases thrombin formation and the activation of oxidative stress, favorably alters fibrin clot properties and may also exert some clinical effects through these mechanisms [30]. The decreased thrombin generation induced by N-3 PUFA may contribute to lower PTL activation and finally to increased effects of antiplatelet drugs. In this setting, the TRAP test used in our study seemed to be important. The test used TRAP-6, a potent agonist which mimicks the PTL-activating action of thrombin. Our findings did not reveal any differences in the TRAP test results after N-3 PUFA supplementation.

\section{Conclusions}

Our results indicate that co-administration of the recommended N-3 PUFA dose does not impair the efficacy of dual antiplatelet therapy measured using WBA in patients with SAP after PCI. Since PTL activation and blood coagulation are interrelated, prospective clinical trials with complex laboratory analyses of the clotting system are necessary in order to evaluate the potential impact of N-3 PUFA on the efficacy of dual antiplatelet therapy as well as on thrombotic events following PCI.

\section{Acknowledgements}

Scientific grant from the Medical University of Silesia.

\section{Conflict of interest: none declared}

\section{References}

1. Fox K, Garcia MA, Ardissino D et al. Guidelines on the management of stable angina pectoris: The Task Force on the Management of Stable Angina Pectoris of the European Society of Cardiology. Eur Heart J, 2006; 27: 1341-1381.

2. Van der Werf F, Bax J, Betriu A et al. Management of acute myocardial infarction in patients presenting with persistent ST-segment elevation: The Task Force on the Management of ST-segment elevation acute myocardial infarction of the European Society of Cardiology. Eur Heart J, 2008; 29: 2909-2945.

3. The ORIGIN Trial Investigators. N-3 fatty acids and cardiovascular outcomes in patients with dysglycemia. N Engl J Med, 2012; 367: 309-318.

4. von Schacky C, Weber PC. Metabolism and effects on platelet function of the purified eicosapentaenoic and docosahexaenoic acids in humans. J Clin Invest, 1985; 76: 2446-2450.

5. Thorngren M, Gustafson A. Effects of 11-week increases in dietary eicosapentaenoic acid on bleeding time, lipids, platelet aggregation. Lancet, 1981; 2: 1190-1193.

6. Nordoy A, Bonaa KH, Sandset PW Hansen JB, Nilsen H. Effect of omega-3 fatty acids and simvastatin on hemostatic risk factors and postprandial hyperlipidemia in patients with combined hyperlipidemia. Arteriosler Thromb Vasc Biol, 2000; 20: 259-265.

7. Vanschoonbeek K, Wouters K, van der Meijden PE et al. Anticoagulant effect of dietary fish oil in hiperlipidemia: A study of hepatic gene expression in APOE2 knock-in mice. Arterioscler Thromb Vasc Biol, 2008; 28: 2023-2029.

8. Barcelli U, Glas-Greenwalt P, Pollak VE. Enhancing effect on dietary supplementation with omega-3 fatty acids on plasma fibrinolysis in normal subjects. Thromb Res, 2006; 118: 307-312.

9. Vanschoonbeek K, Feijge MA, Paquay M et al. Variable hypocoagulant effect of fish oil intake in humans: Modulation of fibrinogen level and thrombin generation. Arterioscler Thromb Vasc Biol, 2004; 24: 1734-1740.

10. Eritsland J, Arnesen H, Seljeflot I, Kierilf P. Long-term effects of n-3 polyunsaturated fatty acids on haemostatic variables and bleeding episodes in patients with coronary artery disease. Blood Coagul Fibrinolysis, 1995; 6: 17-22. 
11. Reis GJ, Boucher TM, Sipperly ME et al. Randomised trial of fish oil for prevention of restenosis after coronary angioplasty. Lancet, 1989; 2: 177-181.

12. Mozaffarian D, Marchiolo R, Gardner T et al. The omega-3 fatty acids for prevention of post-operative atrial fibrillation (OPERA) trial — Rationale and design. Am Heart J, 2011; 162: 56-63.e3.

13. Balk E, Chung M, Lichtenstein A et al. Effects of omega-3 fatty acids on cardiovascular risk factors and intermediate markers of cardiovascular disease. Evid Rep Technol Assess (Summ). AHRQ Publication No. 04-E010-2. Rockville, MD: Agency for Healthcare Research and Quality 2004: 93: 1-6.

14. Wang C, Chung M, Lichtenstein A et al. Effects of omega-3 fatty acids on cardiovascular disease. Evid Rep Technol Assess (Summ), 2004; 93: 1-8.

15. Kristenses SD, Iversen AM, Schmidt EB. n-3 polyunsaturated fatty acids and coronary thrombosis. Lipids, 2001; (26 suppl.): S79-S82.

16. Gajos G, Rostoff P, Undas A, Piwowarska. Effects of polyunsaturated omega-3 fatty acids on responsiveness to dual antiplatelet therapy in patients undergoing percutaneous coronary intervention. J Am Coll Cardiol, 2010; 55: 1671-1678.

17. Gajos G, Zalewski J, Nessler J, Zmudka K, Undas A, Piwowarska W. Polyunsaturated omega-3 fatty acids improve responsiveness to clopidogrel after percutaneous coronary intervention in patients with cytochrome P450 2 C19 loss-of-function polymorphism. Kardiol Pol, 2012; 70: 439-445.

18. Larson MK, Ashmore JH, Harris KA et al. Effects of omega-3 acid ethyl esters and aspirin, alone and in combination, on platelet function in healthy subjects. Thromb Haemost, 2008; 100: 634-641.

19. Gremmel T, Calatzis A, Steiner S et al. Is TRAP-6 suitable as a positive control for platelet reactivity when assessing response to clopidogrel? Platelets, 2010; 21: 515-521.

20. Goodnight Jr, SH, Harris WS, Connor WE. The effects of dietary omega 3 fatty acids on platelet composition and function in man: A prospective, controlled study. Blood, 1981; 58: 880-885.

21. Larson MK, Shearer GC, Ashmore JH et al. Omega-3 fatty acids modulate collagen signaling in human platelets. Prostaglandins, Leucotrienes Fatty Acids, 2011; 84: 93-9.

22. Cohen MG, Rossi JS, Garbarino J et al. Insights into the inhibition of platelet activation by omega-3 polyunsaturated fatty acids:
Beyond aspirin and clopidogrel. Thromb Res, 2011; 128: 335-340 .

23. Guthikonda S, Alviar CL, Vaduganathan M et al. Role of reticulated platelets and platelet size heterogeneity on platelet activity after dual antiplatelet therapy with aspirin and clopidogrel in patients with stable coronary artery disease. J Am Coll Cardiol, 2008; 52: 743-749.

24. Cecchi E, Marcucci R, Paniccia R et al. Effect of blood hematocrit and erythrocyte deformability on adenosine 5'-diphosphate platelet reactivity in patients with acute coronary syndromes on dual antiplatelet therapy. Am J Cardiol, 2009; 104: 764-768.

25. Robier C, Neubauer M, Sternad H, Quehenberger F, Rainer F, Neumeister P. Evaluation of platelet function and pharmacological platelet inhibition in patients with myeloproliferative disorders using multiple electrode aggregometry. Thromb Res, 2010; 126: 232-237.

26. van der Stelt CA, van Werkum JW, Seesing TH, Berg JM, Hackeng CM. To adjust or not to adjust the platelet count in light transmission aggregometry in patients receiving dual aspirin/clopidogrel treatment. Platelets, 2007; 18: 550-553.

27. Lordkipanidze M, Pharand C, Nguyen TA et al. Comparison of four tests to assess inhibition of platelet function by clopidogrel in stable coronary artery disease patients. Eur Heart J, 2008; 29: 2877-2885.

28. Hobson AR, Qureshi Z, Banks P, Curzen NP. Effects of clopidogrel on "aspirin specific" pathways of platelet inhibition. Platelets, 2009; 20: 386-390.

29. Armstrong PC, Dhanji AR, Tucker AT, Mitchell JA, Warner TD. Reduction of platelet thromboxane A2 production ex vivo and in vivo by clopidogrel therapy. J Thromb Haemost, 2010; 8: 613-615.

30. Gajos G, Zalewski J, Rostoff P, Nessler J, Piwowarska W, Undas A. Reduced thrombin formation and altered fibrin clot properties induced by polyunsaturated omega- 3 fatty acids on tpo of dual antiplatelet therapy in patients undergoing percutaneous coronary intervention (OMEGA-PCI Clot). Arterioscler Thromb Vasc Biol, 2011; 31: 1696-1702. 